\title{
TEVAR in aortic dissection: a new standard for Marfan patients during COVID-19?
}

\author{
Amer Harky ${ }^{1}$, Rebecca Abdelmalak ${ }^{2}$, Francesco Torella ${ }^{1}$, and Mark Field ${ }^{1}$ \\ ${ }^{1}$ Liverpool Heart and Chest Hospital NHS Foundation Trust \\ ${ }^{2}$ Imperial College London
}

June 16, 2020

\section{Dear Editor,}

We read with interest the published article by Ikeda et al. [1], they performed thoracic endovascular aortic repair (TEVAR) in a patient with Marfan syndrome (MFS) for acute complicated type B aortic dissection (TBAD) during COVID-19 pandemic.

The evidence around TEVAR for MFS is scarce and open repair remains the gold treatment[2]. During the COVID-19 pandemic, many patients are either being denied treatment or given inferior options on the basis of age, comorbidities and risk of COVID pneumonia; however, the guidelines for aortic intervention in the United Kingdom have remained largely unchanged from pre-COVID-19 era [3]. Our questions to the authors relate to whether their solution was an unnecessary compromise. There is no clear indication defined in their case as a cold leg doesn't necessary means an ischaemic limb. The TEVAR procedure performed aiming to minimise hospital stay, yet this approach may have put the patient at higher risk of developing paraplegia and visceral organ malperfusion, while compromising her long-term care.

There is need to clarify if she had risk factors that prone her to a higher risk acquiring severe COVID-19 which necessitated deviating from the traditional open surgery recommended for MFS patients with TBAD [2]. The authors did not report on renal function, evidence of bowel malperfusion or whether there was resistant hypertension that needed immediate intervention. If the need to expediate intervention was the fear of limb ischaemia, is it conceivable a femoro-femoral bypass could have saved the limb and definitive open surgery on her aorta could have been performed at a later stage, especially since she was haemodynamically stable.

Moreover, as Marfan-diseased aortas are prone to further dilatation, we believe their justification for opting for endovascular repair should also have been more balanced, exploring the know high rate of long-term TEVAR-associated complications in MFS patients including endoleaks, retrograde dissection, stent-graftinduced new entry tears, surgical conversions and reintervention. There is also need for imaging follow-up to assess the success of TEVAR and early detection of aforementioned complications.

\section{References:}

1. Ikeda S, Shih M, Rhee RY, Youdelman BA. Acute complicated type B aortic dissection during the New York City COVID-19 surge. J Card Surg. 2020;1-4.

2. de Beaufort HWL, Trimarchi S, Korach A, et al. Aortic dissection in patients with Marfan syndrome based on the IRAD data. Ann Cardiothorac Surg. 2017;6(6):633-641.

3. Society for Cardiothoracic surgery. SCTS Cardiothoracic practice during Covid-19 outbreak. http://scts.org/SCTS Cardiothoracic practice during Covid-19 outbreak (accessed 08 June 2020). 